\title{
ILCEA
}

Revue de l'Institut des langues et cultures

d'Europe, Amérique, Afrique, Asie et Australie

$12 \mid 2010$

La FASP : dix ans après...

\section{Les mathématiques mises en scène}

Numb3rs entrelacs de cultures

\section{Gwen Le Cor}

\section{(2) OpenEdition}

Journals

Édition électronique

URL : http://journals.openedition.org/ilcea/883

DOI : 10.4000/ilcea.883

ISSN : 2101-0609

Éditeur

UGA Éditions/Université Grenoble Alpes

Édition imprimée

ISBN : 978-2-84310-180-9

ISSN : 1639-6073

\section{Référence électronique}

Gwen Le Cor, « Les mathématiques mises en scène », ILCEA [En ligne], 12 | 2010, mis en ligne le 30 septembre 2010, consulté le 19 avril 2019. URL : http://journals.openedition.org/ilcea/883 ; DOI : 10.4000/ilcea.883

Ce document a été généré automatiquement le 19 avril 2019

(c) ILCEA 


\title{
Les mathématiques mises en scène
}

\author{
Numb3rs entrelacs de cultures
}

\author{
Gwen Le Cor
}

Dans le deuxième volet de sa trilogie, His Dark Materials, Philip Pullman dote un de ses personnages (Will Parry) d'un poignard capable d'ouvrir des fenêtres sur des mondes parallèles. C'est un peu, au fond, au même exercice que se livrent les producteurs d'Ally Mc Beal, Bones ou Wall Street. Du cabinet d'avocat à l'anthropologie médico-légale en passant par l'univers de la finance, les séries télévisées et les scénarios hollywoodiens ouvrent de multiples fenêtres sur les milieux professionnels, nous invitant à suivre le héros dans son monde. À la différence près que là où les mondes de Philip Pullman font appel au rêve et à l'imaginaire, ceux de la fiction à substrat professionnel (FASP) nous plongent dans une représentation d'une profession. Tout à la fois mise en image et cadrage, la FASP nous guide dans un spectacle habillement orchestré du monde professionnel.

1 C'est aussi cela que propose Numb3rs. We all use maths everyday proclame le générique, everything is numbers affirme son héros dans le premier épisode de la série. Nous voilà donc prévenus, Numb3rs est la première série mathématique, et Charlie Eppes son premier héros mathématicien: "television's first mathematical super-hero" (Devlin et Lorden, 2007, Acknowledgments). Telle Alice, le téléspectateur passe donc de l'autre côté du miroir pour s'immerger dans l'univers des nombres et des mathématiques. Mais en s'intéressant aux mathématiques, c'est un saut aussi inhabituel que surprenant que proposent les réalisateurs de la série.

2 Michel Petit lie l'existence de la fiction à substrat professionnel à la «prégnance sociale » du milieu qu'elle décrit, au «niveau de culture populaire dans ces domaines spécialisés » (Petit, 2004, p. 23). «La fiction peut fleurir dans ces différents domaines parce que le juridique, le médical, le militaire paraissent avoir, à des titres divers, une place toujours (plus) importante dans les réalités de la société actuelle»(p.18). Les mathématiques présentent un cas à part. Il est difficile de parler de prégnance sociale des mathématiques. A dire vrai, elles se situeraient plutôt aux antipodes de la culture populaire. Dans l'imaginaire commun, les mathématiques sont une science élitiste et le "matheux » un être plus proche de l'extra-terrestre que de monsieur tout le monde. Aussi inintelligibles 
qu'inaccessibles, «l'espace public leur est interdit» (Sauvageot, 2008, p. 127). Comme le souligne François Sauvageot dans un article consacré au partage du savoir, "les mathématiques sont absentes des revues scientifiques grand public» (Ibid.). Elles sont aussi les grandes oubliées de la fête de la science. De fait, la vulgarisation scientifique privilégie certaines disciplines et, inversement, en censure d'autres, jugées trop théoriques ou trop hermétiques, au rang desquelles figurent les mathématiques.

Inaccessibles au grand public, les mathématiques ne se prêtent pas non plus aisément à la mise en fiction. Mettre les mathématiques en images, donner à voir le langage et les concepts mathématiques, l'idée même semble incongrue. «Considérées comme la science la plus conceptualisée, la plus naturellement dépourvue d'images", la science mathématique est sans doute aussi la "plus difficile à évoquer en film» (Ricco, 1986, p.45). C'est pourtant cela que propose Numb3rs. La série entrelace une fiction commerciale facile d'accès et une science jugée élitiste et hermétique.

Diffusée en prime-time sur CBS depuis 2005, Numb3rs en est à sa $6^{\mathrm{e}}$ saison et compte environ 12 millions de téléspectateurs par épisode (Wolfram Blog). Plébiscitée par le public, la série l'est aussi par les mathématiciens. Gary Lorden, le consultant scientifique de Numb3rs et Keith Devlin, le Math Guy de Weekend Edition', résument bien l'intérêt de la série pour la communauté scientifique :

The gifted actor David Krumholtz has earned the undying love of mathematicians everywhere for bringing Charlie to life in a way that has led millions of people to see mathematics in a completely new light. (Devlin et Lorden, 2007, Acknowledgments)

Véritable coup de projecteur sur une science considérée par beaucoup comme obscure, Numb3rs croise mathématiques et culture populaire.

5 Ma réflexion prend comme point de départ cette étrange intersection entre mathématiques et fiction télévisuelle, pour interroger la représentation des mathématiques à l'écran. Se pose en effet d'emblée la question du statut des mathématiques dans la série: en transposant l'univers mathématique dans l'univers télévisuel, la série opère une translation (au sens mathématique du terme) d'une science (les maths) vers une création artistique et se place de fait au carrefour de deux cultures. Cet article s'attache à cette écriture/réécriture des mathématiques, ainsi qu'aux différentes cultures qui viennent la nourrir.

\section{Porter un nouveau regard sur les mathématiques : vers une option marketing}

Dans un ouvrage consacré aux réécritures littéraires des discours scientifiques, Chantal Foucrier (2006) conçoit la réécriture du discours scientifique par la littérature comme une forme de traduction. Les textes peuvent véhiculer "un certain état des connaissances ", mais ont surtout, selon elle, une fonction de médiation (p. 9). Dès lors, la mise en récit du discours scientifique s'apparente à une sorte de vulgarisation et, de fait, notre problématique rejoint à bien des égards les problématiques soulevés par la vulgarisation, notamment en ce qui concerne le degré de fidélité de la représentation. Et pour les mathématiques, la question est essentielle. En atteste notamment la vision de Baudouin Jurdant (1997). Selon lui, «les mathématiques ne sont pas vulgarisables» car, contrairement aux autres sciences qui peuvent être appréhendées à partir de différents angles, les mathématiques «n'admettent qu'un seul point de vue, celui du mathématicien. Toute autre vision dépossède le contenu de son appartenance au domaine 
des mathématiques»(p.208). Dépouiller un discours mathématique de ses aspects techniques pour en faciliter la compréhension, c'est de fait aussi le dépouiller de sa composante mathématique. Dès lors, les mathématiques vues à travers le prisme de la télévision sont elles encore des mathématiques?

La réception extrêmement favorable de Numb3rs par la communauté mathématique nous apporte un premier élément de réponse. La série fait l'objet d'articles et de conférences placées sous l'égide de la Mathematical Association of America et de la American Mathematical Society: "Working mathematicians of the Mathematical Association of America have recognized the accuracy and validity of the theories and their presentations on the program " (National Science Foundation Press Release n ${ }^{\circ}$ 07-040). Validée par les chercheurs, la série Numb3rs l'est également par les enseignants. Ces derniers utilisent la série comme un outil didactique : le National Council for Teachers of Mathematics est partie prenante dans la création de fiches d'exercices établies à partir de la série et postées sur des sites Web dédiés à Numb3rs (Edmondson, <http://expertvoices.nsdl.org/tvscience /.../>, consulté le 06/10/2009). Les mathématiques à l'œuvre dans la série ont ainsi donné naissance à de nombreux sites universitaires qui démontrent les théorèmes, détaillent et développent les concepts mathématiques mentionnés, soulignent les rares erreurs de présentation ou de calcul et, plus généralement, utilisent l'expertise de mathématiciens pour légitimer l'usage des mathématiques fait dans la série. À titre d'exemple, depuis la première diffusion de la série, Northeastern University lui consacre un blog mensuel ${ }^{2}$ et Wolfram Research détaille les mathématiques utilisées dans les épisodes des saisons 4,5 et 6 , faisant par là même vivre les mathématiques de Numb3rs hors écran ("The MATH behind NUMB3RS » http://numb3rs.wolfram.com/).

7 L'ouvrage, co-écrit par Gary Lorden et Keith Devlin, et qui s'intitule The Numbers Behind Numb3rs: Solving Crime With Mathematics, affiche la même ambition. Au-delà de son soustitre accrocheur (solving crime with mathematics), l'ouvrage s'attache à démontrer la pertinence des concepts mathématiques utilisés dans la série: «Each week, NUMB3RS presents a dramatic story in which realistic mathematics plays a key role in the narrative » (je souligne). À la question de savoir si NUMB3RS donne à voir de vraies maths, les auteurs répondent, sans hésiter, par l'affirmative.

IS THE MATH IN NUMB3RS REAL ?

Both of us are asked this question a lot. The simplest answer is "yes". The producers and writers go to considerable lengths to make sure that any math on the show is correct, running script idea by one or more professional mathematicians from the hundreds across the country that are listed in their address book.

(Devlin et Lorden, 2007, Appendix).

L'ouvrage donne des exemples de cas réels résolus grâce aux mathématiques et remonte aux sources mathématiques des épisodes pour y décrypter les équations et théorèmes à l'œuvre dans la série. La légitimité mathématique de la série ne semble faire aucun doute. Mais au delà de l'intérêt mathématique, les auteurs veulent aussi, et peut-être surtout, répondre aux critiques adressées à la série. Et c'est là un point essentiel. Lors de la diffusion du premier épisode (Jan 23, 2005), les journalistes ont critiqué le manque de vraisemblance de Numb3rs. Comment une équation pourrait-elle permettre de retrouver un tueur en série? Lorden et Devlin s'attachent donc à démontrer la légitimité de la démarche et du modèle utilisés. Ainsi, s'ils détaillent l'équation utilisée dans le premier épisode, ils cherchent surtout à établir qu'elle prend appui sur un cas réel. Ils attestent de la véracité des formules. Les applications mathématiques de la série, aussi improbables soient-elles, sont non seulement possibles mais bien réelles : 
In fact, that entire first episode was based fairly closely on a real case in which a single mathematical equation was used to identify the hot zone where a criminal lived. It was the very equation [...] that viewers saw Charlie write on his blackboard. (p. 5)

Revenons à notre question initiale sur le contenu mathématique de la série. Dans les exemples sus-cités, une partie des mathématiques a lieu hors écran, dans les ouvrages, sites et articles qui lui sont consacrés, un peu comme si, finalement, la série nous montrait la partie émergée de l'iceberg, mais que $90 \%$ de la science était inaccessible au téléspectateur. Mais, s'il n'y a pas de transmission de connaissance, que se passe-t-il pour le téléspectateur?

8 Au-delà de la véracité factuelle, la série est plébiscitée par les mathématiciens parce qu'elle contribue à transformer le regard que le grand public porte sur les mathématiques. Elle est perçue comme un moyen d'une part de dépoussiérer l'image des mathématiques (Zalmanski, p. 41) et, d'autre part, de faire prendre conscience de leur rôle essentiel. Les commentaires abondent en ce sens : "Leave it to television to work out a way to make a national symbol of nerdiness - mathematics - into something sexy " (Hillbery, 2009). D'un point de vue plus théorique, les créateurs de la série, Nick Falacci et Cheryl Heuton, ont reçu un prix en 2007, le National Science Board Group Public Service Award, pour avoir contribué à la compréhension par le public des sciences (public understanding of science). C'est d'ailleurs bien en termes de prise de conscience que peut s'interpréter le générique. Une voix off affirme :

We all use maths everyday: to predict weather, to tell time, to handle money. Maths is more than formulas and equations, it's logic, it's rationality, it's using your mind to solve the greatest mysteries we know.

La série se donne d'emblée pour objet de montrer de façon concrète comment les mathématiques pèsent sur notre vie.

9 Série télévisée et compréhension publique des sciences, on semble plus proche d'une opération de promotion ${ }^{3}$ que d'une véritable peinture du milieu. De fait, les mathématiciens espèrent que Numb3rs fera naître des vocations :

For one thing, successful movies or television series have in the past led to a significant upsurge in the number of students who opt for various majors at university. Perhaps the most cited instance of this is the large number of AI practitioners who were first inspired to enter the field by the 1968 movie 2001 (Devlin 2005, [En ligne], <http://www.maa.org/ devlin/devlin_02_05.html>)

Un peu, au fond, comme si les producteurs avaient pour but secret de médiatiser et redorer le blason des mathématiques, ou pour reprendre la formulation d'Alain Zalmanski, de « mettre les maths à la mode » (2006, p. 40).

Cette approche n'est pas sans poser problème. Les séries télévisées façonnent en effet, à bien des égards, le regard que les téléspectateurs portent sur une profession. Barbara Villez ne dit pas autre chose à propos des séries juridiques. Elle souligne à quel point les séries juridiques participent « à la construction » de la culture juridique (2005, p. 15). Bien qu'il n'y ait «jamais eu de programme systématique, uniforme, d'éducation civique à l'école, » les Américains «connaissent leurs droits et le fonctionnement de leur système juridique»(p.14). Pour elle, cela provient du simple fait d'avoir regardé les séries judiciaires.

11 De la même manière, Numb3rs participe à l'imprégnation mathématique du téléspectateur. Et l'enjeu est de taille. La National Science Foundation mène des études visant à « établir une mesure de l'attitude des citoyens à l'égard de la science et de leur niveau de connaissance » (Bergeron, 1997, p. 186). Les résultats permettent de mesurer 
l'ampleur de la tâche à accomplir puisque «plus de $90 \%$ de la population des États-Unis et du Royaume-Unis est considérée comme scientifiquement illettrée » (Bergeron, p. 186). Se repose donc inévitablement la question de la représentation proposée par la série, à savoir à la fois de l'image transmise et de la logique à l'œuvre.

\section{Du scientifique fictionnel au mathématicien de Numb3rs}

Si les séries judiciaires en sont à leur troisième génération (Villez, 2005, p. 54), Numb3rs écrit la première page des séries mathématiques. Pour autant, Charlie Eppes ne s'inscrit pas sur une page vierge : la représentation des mathématiques dans la série se nourrit des représentations des sciences et des scientifiques qui l'ont précédée.

Avant Numb3rs, le mathématicien au cinéma et à la télévision est le plus souvent un personnage de sexe masculin, déconnecté de toute réalité. Au mieux étrange, c'est souvent la folie qui le caractérise. Les capacités mathématiques hors norme sont toujours synonymes de personnages à la marge. Dans Rain Man, l'autisme du personnage va de pair avec des capacités mathématiques hors du commun, dans Good Will Hunting, le personnage éponyme se révèle incapable de tisser des liens sociaux, et A Beautiful Mind se construit autour de la folie (réelle) de John Nash. En résumé, génie et folie sont étroitement liés dans les représentations des mathématiciens à l'écran : "The second big Hollywood cliché is the instability of the mathematical mind... In Hollywood's world, all mathematicians go mad in the end» (Du Sautoy, 2006, p. 46). Au fond, avant Numb3rs, il y a plus de folie que de mathématiques sur les écrans. Même dans les films consacrés aux mathématiciens, les mathématiques ne constituent au mieux qu'un arrière plan sur lequel se déroule l'intrigue.

13 Numb3rs prend appui sur ces représentations mais s'en écarte aussi d'emblée. En témoigne l'épisode intitulé Prime suspect (saison 1, épisode 5). Le travail de la caméra souligne l'enfermement des mathématiciens dans leur monde. Alternent ainsi des plans consacrés à l'anniversaire d'une petite fille de 5 ans et, en contrepoint, des plans centrés sur le travail de son père, Ethan, sur l'hypothèse de Riemann. Ce dernier est tellement absorbé par son travail qu'il n'entend pas les cris de sa fille et ne se rend pas compte qu'elle est en train de se faire enlever. À bien des égards, la scène évoque un passage de $A$ Beautiful Mind, où l'on découvre un John Nash, absorbé dans ses pensées, qui abandonne son fils dans sa baignoire, le mettant ainsi en danger de noyade. Fait intéressant, Numb3rs ne cautionne pas cette représentation. Le mathématicien qui délaisse son enfant n'est pas le héros mais un personnage secondaire dont la découverte peut amener le chaos financier. Son travail sur l'hypothèse de Reimann peut donner le moyen de décrypter tous les codes et donc ouvrir aux criminels l'accès à tous les comptes et toutes les transactions financières. Ethan représente le côté sombre hérité de la tradition littéraire gothique $^{4}$, alors que Charlie Eppes contribue, au contraire, à déconstruire le stéréotype, nous y reviendrons.

14 La question de la représentation des mathématiques dans Numb3rs est indissociable des mythes et stéréotypes relayés par les fictions qui l'ont précédée. De la même manière, elle ne peut s'appréhender sans aborder la question du genre. Classée dans les police procedurals, Numb3rs est une fiction policière mathématique, " amath-based, crime-solving drama » selon les termes du site de la National Science Foundation. Objet fictionnel, Numb3rs 
est aussi un objet télévisuel. Sa place dans la grille de programmation, en prime-time, et le réseau (network) CBS (Columbia Broadcasting System) qui la diffuse, contribuent aussi à caractériser la série. La série, nous allons le voir se nourrit de toute une culture télévisuelle.

\section{Numb3rs, pur produit télévisuel ?}

15 Comme le rappelle Marie-France Chambat-Houillon à propos des chaînes françaises, «le succès d'une série s'explique en partie par la relation crédible que noue la châ̂ne avec son émission. Autrement dit, le succès peut s'expliquer par les façons dont une série policière devient série propre à une chaîne ou a une autre » (Beylot et Sellier, 2004, p. 85) ${ }^{5}$. Crime Scene Investigation (CSI) ou dans sa version française Les Experts, et ses spin-offs (Miami et $\mathrm{NY}$ ), sont l'ancrage de CBS, sa signature. Diffusée sur le même réseau (network) que CSI, Numb3rs s'inscrit donc d'emblée dans son sillage. Surfant sur la vague CSI, la série suit la même recette d'une série à succès et en propose une variante mathématique où l'expertise mathématique a remplacé l'expertise médico-légale.

16 À bien des égards en effet, le schéma et la structure de Numb3rs sont hérités des Experts. À l'inverse de séries comme Ally Mc Beal qui fonctionnent sur le principe de récits ouverts, les épisodes de Numb3rs et CSI sont autonomes et le problème (ou le crime) est systématiquement résolu en un épisode. Le principe est attesté : la science (expertise médico-légale ou mathématiques) est le protagoniste principal dans la lutte contre le crime et, en bon héros, elle triomphe toujours. L'autonomie des épisodes renforce encore l'impression de toute puissance de la science. D'ailleurs, contrairement aux enquêtes réelles, les criminels de Numb3rs, à l'instar de ceux de CSI, laissent toujours des indices pour être démasqués. Selon une logique similaire, Charlie Eppes tient plus du super-héros mathématique que du mathématicien lambda. Il maîtrise toutes les théories mathématiques, parvient à réunir toutes données nécessaires en un temps record et résout bien entendu tous les problèmes à l'aide d'équations mathématiques.

On retrouve dans Numb3rs le même découpage narratif que dans CSI. Là où CSI alterne séquences d'enquête sur le terrain et travail de laboratoire, Numb3rs alterne enquête sur le terrain et séquences mathématiques. À titre d'exemple, l'épisode 11 de la saison 1 (intitulé Sacrifice) commence par une scène de crime qui n'a rien à envier à CSI. La seule différence étant peut-être que le cadavre que Don Eppes découvre est celui d'un informaticien. Dans l'enchaînement des séquences, la scène de crime fait place au générique. La caméra nous entraîne alors dans les bureaux du FBI pour une explication scientifique sur les codes, avant de se recentrer sur une scène d'interrogatoire. On retrouve la même alternance de séquences et la même mise en scène rapide que dans Les Experts. Les conventions narratives et la trame sont bien celles de CSI : policiers dépêchés sur la scène de crime, scènes d'interrogatoires, scène de laboratoire.

18 Sur le plan visuel, Numb3rs a tout d'une série dérivée de CSI. Une des raisons du succès de CSI tient à la manière dont la série donne à voir les explications scientifiques dans des plans en forme de retour-en-arrière qui retracent les conditions initiales du crime. Le téléspectateur peut ainsi suivre la trajectoire de la balle, la voir transpercer la peau et les organes de la victime avant, par exemple, de se loger dans un mur. De la même manière, Numb3rs donne vie aux explications de Charlie Eppes. Ses développements sur les codes, sur la chaîne de Markov, ou sur le fonctionnement des statistiques, apparaissent à l'écran 
dans des cadres qui se superposent à l'image initiale. Dans les deux cas, les codes de couleurs fonctionnent de la même manière. Les couleurs des explications et retours-enarrière sont atténuées et jaunies par rapport aux couleurs vives des actions présentes.

Le rapprochement entre les deux séries va plus loin encore: Numb3rs s'inspire directement des scénarios des séries policières. Le premier épisode en atteste: Charlie utilise une technique de profilage géographique (Criminal Geographic Targeting) pour retrouver un tueur en série, à la différence près qu'au lieu d'utiliser un profilage psychologique, Charlie se base sur une équation mathématique pour délimiter la zone où le tueur a la plus grande probabilité de se trouver.

Enfin, le scénario de Numb3rs suit les conventions narratives traditionnelles. Il se construit sur une opposition entre deux personnages, deux frères, qui incarnent deux approches antithétiques. La vision abstraite de Charlie répond ainsi à celle, plus pragmatique, de Don, l'approche théorique du premier s'opposant à l'approche pratique de son frère, approche héritée d'une expérience du terrain (Devlin et Lorden, 2007, p. 209). Don joue aussi le rôle du profane, de celui à qui Charlie doit expliquer ses théories. Cette opposition fait naître un jeu de questions/réponses qui permet d'expliciter le vocabulaire scientifique et de détailler des concepts mathématiques trop hermétiques pour le téléspectateur. Numb3rs utilise là un ressort habituel de l'écriture des séries télévisées, et Don Eppes rejoint en cela toute une galerie de personnages qui servent de prétextes à une explication scientifique. C'est le cas notamment de l'agent Seeley Boothe de Bones, un autre agent du FBI, imperméable à la terminologie scientifique.

Numb3rs est un objet télévisuel, cela ne fait aucun doute, mais cette définition suffit-elle à définir la série ? On pourrait être tenté de la réduire à son appartenance générique aux séries policières (police procedurals), à ne voir qu'un «imaginaire» mathématique, pourtant les mathématiciens en ont une autre lecture. Et c'est bien là tout l'intérêt.

\section{Culture mathématique}

22 La réponse ne se situe ni du côté de la véracité factuelle, ni du côté du langage mathématique employé, mais bien du côté de la culture. En témoigne notamment le point de vue de Jonathan Farley, un professeur de mathématiques de Harvard, qui est à l'origine d'une compagnie d'expertise mathématique et scientifique pour les films et la télévision (Hollywood Math and Science Film Consulting). J. Farley décrit son rôle de consultant scientifique en termes culturels: "It is not just about fixing mathematical mistakes in the script... It is also about helping them get the culture right » (Kunzelman, 2005).

Dans un autre domaine, celui de la chimie, Carl Djerassi, un chimiste renommé qui s'est tourné vers l'écriture, dit s'être consacré à l'écriture pour contribuer à la compréhension de la culture scientifique :

Because I wanted to contribute beyond PUS ("public understanding of science")... by using the tools of the humanist rather than the scientist to illuminate the latter's culture for a broader audience. (Djerassi, 2005, p. 181)

C'est sans doute sous cet angle que Numb3rs est le plus intéressant. La série peut être appréhendée comme peinture d'un milieu, comme fenêtre ouverte sur une culture mathématique. C'est cette dernière fenêtre qu'il nous faut maintenant ouvrir.

Le socio-anthropologue Dominique Géraud parle de «clans ou tribus de la communauté scientifique » (Fabre et Mustière, p. 65). Carl Djerassi ne dit pas autre chose lorsqu'il fait 
référence aux tribus scientifiques ou lorsqu'il définit la science comme une communauté dans Cantor's Dilemma : "Science is both a disinterested pursuit of truth and a community, with its own customs, its own social contracts. » Numb3rs dévoile la tribu mathématique ${ }^{6}$ au grand public, la met en scène. Dans ce théâtre des mathématiques, la première étape est sans doute le décor. Comme le rappellent à la fois le blog de Wolfram et un article de Cal Tech News, le rôle de consultant scientifique de la série consiste d'une part à vérifier l'authenticité mathématique, et d'autre part à fournir l'arrière plan mathématique de la série :

In addition to technical consultation for the writers and coaching for the actors, Wolfram research helps dress the set.

[En ligne], <http://blog.wolfram.com/2007/...>.

Besides reviewing scripts for mathematical authenticity, he has also been asked to come up with math or physics concepts and equations that provide the "mathematical background to what some of the characters are doing, saying, or thinking. This could include pictures or things to write on notepads that the camera might see, or stuff that Charlie writes on a blackboard or whiteboard."

[En ligne], <http://pr.caltech.edu/periodicals/...>.

L'arrière plan mathématique, ce sont d'abord les équations et lignes de calculs. Du tableau noir au tableau blanc en passant par les ordinateurs ou par des écrans transparents aux effets des plus cinématographiques, les équations envahissent la scène. Au-delà de l'effet décoratif, c'est une fenêtre ouverte sur une culture. Dans le deuxième épisode par exemple, afin d'assurer la cohérence mathématique des équations et la rapidité d'écriture nécessaire, les producteurs ont eu recours à un doublage de la main de l'acteur lors des gros plans sur les équations. Leur écriture a ainsi été confiée à la main experte d'un doctorant en mathématiques de $\mathrm{Cal} \mathrm{Tech}$.

Une fois le décor mis en place, se pose alors la question du/des milieu(x) mis en fiction. Les mathématiques sont multiples: des mathématiques pures aux mathématiques appliquées, la palette de tribus possibles est large. La série opère des choix : si elle offre un aperçu des différentes branches des mathématiques, elle présente avant tout les mathématiques comme une science de la modélisation. En annonçant dès le générique " We all use maths everyday", la série affirme la pertinence des modèles mathématiques sur notre vie. Plusieurs raisons peuvent expliquer ce choix.

La première explication est d'ordre purement pratique: le premier consultant mathématique de la série étant un statisticien, la série s'oriente naturellement vers les statistiques et les probabilités. Ce choix peut aussi s'envisager comme une forme de réponse aux attentes des téléspectateurs. Dans l'opinion publique, la science se réduit souvent à ces applications, et dans Numb3rs il est surtout question de nombres et de mathématiques appliquées. Même lorsque la série fait référence à des concepts appartenant au domaine des mathématiques pures, comme par exemple l'hypothèse de Riemann, c'est pour en considérer une de ses applications: son utilisation en cryptanalyse (Saison 1, épisode 5, Prime Suspect).

Sans doute enfin faut-il y voir une empreinte culturelle anglo-saxonne. L'école française et l'école russe des mathématiques sont davantage basées sur le formalisme, tandis que l'école américaine laisse une plus grande part à la modélisation. Les statistiques et les probabilités ont notamment une place plus importante dans l'enseignement des mathématiques aux États-Unis qu'en France.

La représentation offerte s'appuie donc à la fois sur des schémas culturels préétablis et sur des contraintes liées aux choix d'expertise, mais pour autant elle expose les 
mathématiques comme jamais aucun film ou série ne l'avait fait auparavant. Et c'est bien là le point essentiel. Comme le soulignent Devlin et Lorden, Numb3rs est une série mathématique parce qu'elle donne à voir un authentique cheminement mathématique :

The one accurate thing that we believe comes across in practically every episode is the approach Charlie brings to the problems Don presents him. (Devlin et Lorden, 2007, p. 208)

Quelle est donc cette démarche mathématique?

Parmi les clichés sur les mathématiques, le plus tenace est sans doute celui d'une approche basée sur un cheminement aussi logique que froid. Les mathématiciens soulignent pourtant tous l'importance de l'intuition et de l'imagination dans la démarche mathématique. Terence Tao, l'un lauréats de la dernière médaille Fields en $2006^{8}$, pense «que la chose la plus importante pour développer l'intérêt pour les mathématiques est d'avoir la capacité de jouer avec elles " (Busser, 2006, p. 15). Numb3rs nous donne à voir cet esprit mathématique empreint de jeu, souligne l'importance de l'imagination et de l'intuition dans le cheminement mathématique. Le premier épisode est symptomatique. Charlie tente de modéliser le comportement d'un tueur en série dans l'espoir de parvenir à sauver la prochaine victime. Il trouve la solution au problème, non pas un crayon ou une craie à la main, mais en observant un système d'arrosage de pelouse. Il établit alors une analogie entre la chute aléatoire des gouttes sur la pelouse et le hasard qui semble présider au choix des victimes. Il explique à Don qu'aucun modèle mathématique, aussi élaboré soit-il, ne peut permettre de prédire avec exactitude l'endroit où tombera la prochaine goutte. Cependant, en observant la distribution des gouttes sur la pelouse, il est possible de remonter à sa source afin de découvrir l'emplacement du système d'arrosage. Charlie se base alors sur cette intuition pour élaborer un modèle mathématique de profilage géographique qui lui permet de cibler la zone où vit le tueur.

Cette approche quasi-expérimentale des mathématiques, que l'on retrouve dans nombre d'épisodes, doit sans doute aussi beaucoup à l'approche scientifique anglo-saxonne. Le cheminement scientifique anglo-saxon fait la part belle à l'expérimentation'. Aux ÉtatsUnis l'enseignement des mathématiques prend comme point de départ la manipulation et l'expérimentation, alors qu'en France il s'appuie davantage sur les concepts et théorèmes.

\section{Cheminement mathématique et esthétique}

Numb3rs s'attache aussi au cheminement mathématique lui même. La série montre la frustration qu'éprouve Charlie face aux problèmes soulevés, nous donne à voir ses tâtonnements pour parvenir à la solution. Elle présente, sommes toutes, ce que Laurent Schwarz, lauréat de la médaille Fields en 1950, appelle les zigzags du cheminement. Laurent Schwartz dit qu'il « cherche en zigzag et arrive finalement plus près du point de départ qu'il ne l'avait pensé. » Il éprouve alors le besoin de trouver un autre chemin, plus court, pour arriver au résultat " (Fabre et Mustière, 2008, p. 135). Cette nécessité d'aller au plus court, au plus beau, se retrouve aussi dans Numb3rs. Comme le souligne le physicien de la série dans le premier épisode, "Charles, you are a mathematician, you are always looking for the elegant solution. Human behavior is rarely if ever elegant, perhaps you need your equation to be less elegant. »

31 Ce dernier point mérite notre attention. Rien n'est plus étranger, a priori, pour le «non matheux » que mathématique et esthétique, et pourtant les mathématiciens se délectent de l'élégance d'une démonstration, de son esthétique. Dans un ouvrage intitulé 
Mathématiques et Logiques, les auteurs soulignent le lien entre cheminent mathématique et esthétique «les mathématiques sont une science, c'est également un art. Le critère de jugement des mathématiques est toujours esthétique, tout au moins en partie » (Kac et Ulam, 1973, p. vi). Elégance, cohérence et concision d'une démonstration sont toujours synonymes de beauté.

Charlie est convaincu par la beauté de ses équations, cela ne fait aucun doute, mais Numb3rs utilise aussi le medium télévisuel pour transposer la beauté sur le plan artistique. Dans le premier épisode, la caméra zoome sur un tableau noir, Amita, une doctorante en mathématiques promène lentement et sensuellement sa main sur les équations qui y sont inscrites. Le tableau est alors baigné d'une lumière scintillante qui donne une impression de magie et de beauté, impression renforcée par la musique qui accompagne la scène. En mettant en scène cette équation, c'est la beauté des mathématiques que les producteurs nous donnent à voir.

Numb3rs se présente finalement comme une invitation au voyage dans un monde mathématique. Mais loin de faire écran à la science, la série nous la donne à voir. Pour Denis Guedj, mathématicien et auteur d'une fiction mathématique, Le Théorème du Perroquet, l'image et le son permettent d'appréhender des concepts impossibles à saisir autrement. Il voit dans le son un allié irremplaçable pour saisir l'infini : «Pour suggérer l'infini, par exemple. Sur une feuille ou sur un écran, finis tous les deux, on ne peut représenter une droite, infinie ; l'image ne nous offre que des segments. Un son qui se perd dans le lointain peut faire l'affaire » (Guedj, 1994, p. 221).

En présentant des mathématiques synonymes de beauté, d'intuition, et de jeu, Numb3rs se situe aux antipodes de la vision caricaturale de la rigueur froide de cette science. La série humanise le mathématicien, et lui permet d'entrer dans le monde du grand public. Pour autant, la représentation qu'elle propose reste fidèle à la démarche mathématique. La FASP mathématique de Numb3rs fonctionne alors comme une passerelle reliant deux univers. Et c'est peut-être là son intérêt essentiel.

La spécialisation croissante des sciences, a eu pour conséquence directe de creuser l'écart entre le spécialiste et le non-spécialiste. Mais cette incompréhension mutuelle a aussi ouvert la voix à de nouveaux modes de communication entre spécialistes et nonspécialistes.

This process of professionalization, autonomization and disentanglement from both the public and the general culture has been accompanied by the creation of new channels of communication between specialists and non-specialists.

(Bucchi, 1998, p. 1)

Massimiano Bucci fait ici référence aux expositions, conférences, musées, manifestations et articles de vulgarisation, auxquels on pourrait rajouter les manifestations scientifiques du type «Fête de la science». Mais je pense que c'est également ainsi que peut se comprendre la FASP mathématique. Elle est née d'un besoin: celui de comprendre le monde mathématique. Elle s'apparente en cela à d'autres formes de communication récentes que sont les « bars des sciences » ( Cafe scientifique » en anglais) créés à la fin des années 1990, avec comme but de faire vivre la science en dehors d'un contexte universitaire ${ }^{10}$.

Numb3rs est une création artistique télévisuelle, mais il ne faut pas y voir là un appauvrissement de la science, bien au contraire. La série s'appuie sur la culture télévisuelle des spectateurs pour mettre la culture mathématique anglo-saxonne en scène. 


\section{BIBLIOGRAPHIE}

BEYLOT, Pierre et Genevièvre Sellier (dir.), Les séries policières. Les medias en Actes. Paris,

L'Harmattan, 2004, 405 p.

BERGERON, Andrée, « La culture scientifique des scientifiques. » dans La promotion de la culture scientifique et technique: ses acteurs et leurs logiques, Publications de l'université Paris 7 - Denis Diderot, 1997, p. 185-200.

BUCCHI, Massimiano, Science and the Media : Alternative Routes in Scientific Communication, Routledge Studies in Science, Technology, and the Society, 1998, 198 p.

BUSSER, Elisabeth, «Terence Tao et Andrei Okounkov : les deux autres Fields », Tangente $\mathrm{n}^{\circ} 112$ (sep-oct 2006), p. 14-16.

CafeScientifique, Disponible sur <http://www.cafescientifique.org/>, consulté le 06/10/2009.

DEVLIN, Keith et LORDEN Gary, The Numbers behind NUMB3RS: Solving Crime with Mathematics, New York, A Plume Book, 2007, 243 p.

DEVLIN, Keith, « NUMB3RS gets the math right », Devlin's Angle dans MAA Online, February 2005, disponible sur <http://www.maa.org/devlin/devlin_02_05.html>, consulté le 06/10/2009.

DJERASSI, Carl, « Science-in-Fiction: Literary Contraband », Dans Peter FREESE and Charles B. HARRIS (dir.), Science, Technology and the Humanities in Recent American Fiction, Essen, Verlag Die Blaue Eule, 2004, p. 181-201.

-, Cantor's Dilemma. New York : Doubleday, 1989.

DUPERAY, Max, Lectures de Frankenstein, Presses universitaires de Rennes,1997, 112 p.

DU SAUTOY, Marcus, New Scientist, April 1, 2006, p. 46.

EDMONDSON, Brad, « "Numb3rs” Honored by NSF » May 5th, 2007. Disponible sur <http:// expertvoices.nsdl.org/...>, consulté le 06/10/2009.

FABRE, Michel et Philippe Mustière (dir), Rencontres Jules Vernes : Le partage du savoir. Nantes, Coiffard libraire, 2008, $403 \mathrm{p}$.

FOUCRIER, Chantal (dir.), Les réécritures littéraires des discours scientifiques. Paris, Michel Houdiard Editeur, 2006, 375 p.

GRÜNZWEIG, Walter, « Science-in-Fiction: Science as Tribal Culture in the Novels of Carl Djerassi » dans Peter FREESE and Charles B. HARRIS (dir.), Science, Technology and the Humanities in Recent American Fiction, Essen, Verlag Die Blaue Eule, 2004, p. 231-248.

GUEDJ, Denis, «Les fictions scientifiques au cinéma », dans MARTINET, Alexis (dir.), Le cinéma et la science, CNRS éditions, 1994, p. 218- 223.

HILLBERY, Rondha, «Crime and Computation », Caltech News, vol. 39, n 1, disponible sur <http:// pr.caltech.edu/periodicals/...>, consulté le 06/10/2009.

JURDANT, Baudouin, « Enjeux et paradoxes de la vulgarisation scientifique » dans La promotion de la culture scientifique et technique : ses acteurs et leurs logiques, Publications de l'université Paris 7 - Denis Diderot, 1997, p. 201-209. 
KAC, Mark et Stanislaw Ulam (dir.), Mathématiques et Logiques : rétrospective et perspectives, [Traduit par Philippe Gatbois], Paris, Dunod, 1973, 178 p.

KUnZeLman, Michael. « Harvard Professor offers services as Hollywood mathematician. » Boston.com News. May 1, 2005, disponible sur < http://www.hollywoodmath.com/

AP_Boston_Globe_I. pdf>, consulté le 06/10/2009.

National Science Foundation Press Release \# 07-040, April 16, 2007, « The "Numb3rs" Add Up: Popular TV Show and Its Creators Receive Public Service Award, disponible sur <http://www.nsf.gov/ news/...>, consulté le 06/10/2009.

Numb3rs Blog. Northeastern University, disponible sur <http://nuweb2.neu.edu/math/cp/blog>, consulté le 06/10/2009.

PETIT, Michel, « Quelques réflexions sur la fiction à substrat professionnel » dans PETIT, Michel (dir.) et ISANI, Shaeda (collab.), Aspects de la fiction à substrat professionnel, Université Victor Segalen Bordeau 2, coll. « Travaux 20.25»,2004, p. 3-24.

PONNAU, Gwenhaël, « Aspects du discours fictionnel de la science » dans FOUCRIER, Chantal (dir.), Les réécritures littéraires des discours scientifiques, Paris, Michel Houdiard Editeur, 2006, p. 286-298. PREDAL, René, « Le savant et la science dans le cinéma et la fiction. » La science à l'écran. Cinémaction $\mathrm{n}^{\circ} 38,1986$, p. 162-173.

RIcco, Georges, «Images et mathématiques », La science à l'écran. Cinémaction n 38, 1986, p. 45-51. SAUVAGEOT, François, «Les yeux d'Uranie : le regard et les mots des mathématiciens » dans FABRE, Michel et MUSTIÈRE, Philippe (dir.), Rencontres Jules Vernes : Le partage du savoir, Nantes, Coiffard Libraire, 2008, p. 127-139.

SUlLIVAN, J. W. N. « Mathematics as an art », dans SCHAAF, William L. (dir.), Mathematics our great heritage: Essays on the nature and cultural significance of Mathematics, New York, Harper and Brothers, 1948, p. 3-13.

VILLEZ, Barbara, Séries télé: Visions de la justice. Presses universitaires de France, 2005, 193 p.

Wolfram Blog « The MATH behind NUMB3RS », disponible sur <http://numb3rs.wolfram.com/>, consulté le 06/10/2009.

WYNNE, Marcus, «Wolfram Research goes to Hollywood » Wolfram Blog September 25, 2007, disponible sur <http://blog.wolfram.com/2007/09/25/wolfram-research-goes-to-hollywood>, consulté le 06/10/2009.

ZALMANSKI, Alain « Numb3rs », Tangente, $\mathrm{n}^{\circ} 110$ (Mai-juin 2006), p. 40-41.

\section{NOTES}

1. Outre son rôle de consultant mathématique pour Weekend Edition, (diffusée par le National Public Radio), Keith Devlin est "consulting professor » au département de mathématiques de Stanford University depuis 2001.

2. "This is a blog where a professor from Northeastern University's Math department posts mathematical comments on the television show Numb3rs » [En ligne], <http://nuweb2.neu.edu/math/cp/blog/>.

3. L'impression est renforcée par l'existence de sites web qui, tout en étant dédiés à Numb3rs, sont sponsorisés par deux concepteurs de logiciels de calcul formel, à savoir Texas Instrument et Wolfram (Wolfram commercialise le logiciel « mathematica »). 
4. Le cinéma et la télévision ne nous donnent d'ailleurs que très peu de représentations de mathématiciens. L'origine de l'image du mathématicien qui fascine autant qu'il dérange est donc à chercher ailleurs, dans les représentations inquiétantes des scientifiques et de la science dans la fiction. On peut remonter ainsi jusqu'à la première occurrence du savant en littérature, le Dr Frankenstein de Mary Shelley (1818). Chercheur passionné qui se jette à corps perdu dans sa recherche, Victor Frankenstein (« he felt like a child picking up shells beside the great and unexplored ocean of truth » Frankenstein, p. 39) incarne l'aspect sombre de la curiosité scientifique, celle du scientifique qui transgresse les limites. Sa création est « imbriquée dans le mal » (Duperay, 1997, p. 86), et la fascination pour le pouvoir de la science se traduit dans l'image du scientifique incontrôlable. En franchissant toutes limites, le Dr Frankenstein « remet en cause son humanité » (p. 85). Le scientifique de fiction doit beaucoup à cette filiation. Du récit gothique et récit d'épouvante, on garde l'image de l'inhumanité des scientifiques, de leur folie. À l'écran, le scientifique est tout aussi inquiétant. Comme le souligne René Prédal dans un numéro de Cinémaction consacré à La science à l'écran : «Il faut bien se rendre à l'évidence : la seule image de savant (docteur ou professeur) qui ait vraiment marqué l'histoire du cinéma est celle du savant fou » (1986, p. 163). Du Dr Frankenstein en passant par le Dr Moreau ou le Dr Jekyll, le scientifique est celui qui ouvre la boite de Pandore.

5. L'article s'attache aux enjeux communicationnels et à la manière dont les chaînes s'approprient un programme.

6. Il faut entendre tribu au sens sociologique et non au sens mathématique du terme (analyse).

7. «Graduate student David Grynkiewicz worked as a hand double in early Numb3rs episodes. David Krumholtz as Charlie grapples with an equation in a scene filmed in Caltech astronomy professor Nick Scoville's office. »[En ligne]<http://pr.caltech.edu/periodicals/CaltechNews/articles/v39/ crime.html>.

8. La médaille Fields est décernée tous les quatre ans.

9. Ces différences culturelles sont à l'origine du projet de « La main à la pâte » lancé à l'initiative Georges Charpak (prix Nobel de Physique). Son initiative est née de l'observation du projet américain « Hands On ».

10. Cafe Scientifique is a place where, for the price of a cup of coffee or a glass of wine, anyone can come to explore the latest ideas in science and technology. Meetings have taken place in cafes, bars, restaurants and even theatres, but always outside a traditional academic context. The first Cafes Scientifiques in the UK were held in Leeds in 1998. From there, cafes gradually spread across the country. Currently, some forty or so cafes meet regularly to hear scientists or writers on science talk about their work and discuss it with diverse audiences. [En ligne], <http://www.cafescientifique.org/>.

\section{RÉSUMÉS}

En associant mathématiques et fiction commerciale à grand succès, la série Numb3rs se situe à l'intersection de la culture mathématique et la culture télévisuelle. L'article explore cette intersection, et interroge la représentation des mathématiques qui nous est donnée à voir. Il s'attache dans un premier temps à la réception très favorable de la série par le milieu mathématique anglo-saxon. L'auteur analyse ensuite les caractéristiques héritées de la culture télévisuelle (codes narratifs, schéma, structure). Enfin, l'article a pour ambition de montrer que 
Numb3rs est une fenêtre ouverte sur la culture mathématique anglo-saxonne, de montrer que la série permet d'appréhender la démarche et le cheminement mathématique anglo-saxonne.

Numb3rs weaves popular entertainment and mathematics to an unprecedented extent, thus placing the show at a crossroads of mathematical culture and television culture. The impulse behind this paper originates in this very intersection, in the attempt to analyse the way mathematics is represented in the show. The paper first focuses on the positive reactions of the Anglo-Saxon mathematical community to the mathematics presented in Numb3rs. It then examines the narrative codes and structure, and shows that they are directly inherited from the genre of popular television drama. Finally, this paper aims at showing that the fictional representation is an open window onto Anglo-Saxon mathematics culture. It allows the viewers to apprehend the Anglo-Saxon mathematical approach and reasoning.

\section{INDEX}

Keywords : Anglo-Saxon mathematics culture, Numb3rs, TV series, Public understanding of science, Representation, Anglo-Saxon mathematical approach

Mots-clés : culture mathématique anglo-saxonne, Numb3rs, séries télévisées, compréhension publique des sciences, représentation, démarche mathématique anglo-saxonne

\section{AUTEUR}

\section{GWEN LE COR}

Université de Paris 8

Laboratoire Suds d'Amérique 